\title{
Distributed Ledger Technology for the systematic Investigation and Reduction of Information Asymmetry in Collaborative Networks
}

\author{
Markus Schinle \\ FZI Research Center for \\ Information Technology \\ schinle@,fzi.de
}

\author{
Christina Erler \\ Karlsruhe Institute of \\ Technology \\ christina.erler@kit.edu
}

\author{
Wilhelm Stork \\ Karlsruhe Institute of \\ Technology \\ wilhelm.stork@kit.edu
}

\begin{abstract}
Costs, risks and inefficiencies in Collaborative Networks (CNs) resulting from information asymmetries have been discussed in the scientific community for years. In this work, supply chain networks, as common representative of CNs, are used as object of investigation. Therein, problems and requirements of interorganizational information exchange are elaborated as well as the potential role Distributed Ledger Technology (DLT) could play to address them. As major challenge, convincing all relevant network partners to resolve asymmetric information by sharing sensitive data is identified. To face this issue, the value of shared information is prioritized as a motivational aspect. Finally, we propose a search process to systematically assess the benefits of information sharing in collaborative networks. To coordinate and implement this process regarding the derived requirements of CNs we propose system components based on DLT design patterns.
\end{abstract}

\section{Introduction}

Various approaches are currently being discussed as to how Distributed Ledger Technology (DLT), in particular Blockchain, could be used in business applications. Blockchain became famous for the enablement of immutable and secure transactions within a peer-to-peer network without the need for additional trusted intermediaries [1]. The advantages of improved data integrity, decentralization, disintermediation and thus reduced transaction costs are also seen as an advantage of DLT-based information system components in different kinds of applications [2]. In summary, profit is seen for the individual players in the network by having the opportunity to access data which otherwise would not be in their scope [2-5]. Particularly in the case of specific CNs like supply chains, it was

URI: https://hdl.handle.net/10125/64393

978-0-9981331-3-3

(CC BY-NC-ND 4.0) found that information sharing and coordination helps to reduce the bullwhip effect and the supply chain costs $[31,32,42]$. Therefore, DLT could enable innovative business models as well as new quality of collaboration in business networks. Nevertheless, adoption of this technology is seen as a long lasting process over years [6]. With this work, we want to contribute to understanding and leveraging DLTs' potential for the investigation and reduction of information asymmetry in CNs.

\section{Basics}

Different types of DLT have evolved so far [7]. While public distributed ledgers are accessible to anybody, permissioned distributed ledgers require authentication and authorization within a consortium network. An approach to construct modular permissioned architectures for Blockchains are so-called sidechains $[7,8]$. The architecture consists of a central consortium Blockchain and a set of private subnets. Access requests are managed via the consortium Blockchain. The subnets are used for local transactions to share information between groups of partners. Therein a local transaction only requires consensus between the nodes associated with the partners. With the sidechain approach, data can be hidden from other competitors and can only be exchanged with a trusted circle of partners. A sidechain can therefore provide more privacy within a consortium network for example in business applications [7]. Xu et. al. identifies and describes design patterns for Blockchain-based applications [2]. In this work, we use the following three of these patterns. The reverse oracle pattern is used to interact with the external world - it can be used from existing off-chain components to get on-chain data and verify if required conditions are met. As second pattern, we introduce encrypting on-chain data. It is classified as a data management pattern and used to hide sensitive on-chain data to specific participants with encryption. Also, tokenization is part of this class. It is used to 
represent transferable assets like currency. Solutions based on DLT may have different application fields. In our case, we try to apply it on coordination processes within collaborative networks. To get a common understanding of this term we use the definition of Camarinha-Matos and Afsarmanesh [9]. They define collaborative networks as "constituted by a variety of entities (e.g. organizations and people) that are largely autonomous, geographically distributed, and heterogeneous in terms of their: operating environment, culture, social capital, and goals. Nevertheless these entities collaborate to better achieve common or compatible goals, and whose interactions are supported by computer network" [9].

\section{Research Challenge and Question}

Supply chains are mentioned as common example for CNs in literature, which is why we have also selected them as the subject of our investigation $[10,11]$. Therein, more transparency and reduction of asymmetric information is seen as needed, satisfiable through improved information exchange and coordination. So far, reasons why the operation of common systems to satisfy this need in CNs often fails are on different levels e.g. by economical, organizational and technical implications (see section 5). Therefore, we first elaborate the existing problems regarding the lack of information exchange in such collaborative networks and derive the requirements for improved information exchange (see section 5.1).

In recent years, Supply Chain Management (SCM) has also emerged as an interesting application field for DLT as its default state of truth is seen as an opportunity to improve interorganizational information exchange [24]. Between most of the involved parties, there are various reasons for that e.g. to demonstrate sustainability of products, to coordinate product recalls or to reduce bullwhip effects. One can also observe the opportunity to expand the current optimization potential of processes from internal to interorganizational level with process management based on DLT [5]. That is why we assume that the reduction of information asymmetry can serve as the central feature and the pivot for the decision about the use of this technology as base of an interorganizational information system. To leverage the mentioned opportunities and keep the promises of a decentralized and distributed system, different stakeholders in CNs need to join a collaborative system. As voluntary information exchange between organizations need to be beneficial for all of them [12], the main question of this work is how the search process for a win-win situation can be coordinated and implemented.

\section{Methodology}

For our research, we used the Design Science Research Methodology (DSRM). According to Peffers et al., the approach can be used to solve problems at the intersection of IT and organizations [13]. Due to this fact and the primary goal to develop a new artifact in the form of a coordination process for the reduction of information asymmetry in CNs, the approach of Peffer et al. is preferred to other existing DSRM approaches $[14,15]$. The DSRM consists of the following six process steps: (1) Problem identification and motivation, (2) Definition of objectives of solution, (3) Design and development of the solution artifact, (4) Demonstration of the solution artifact, (5) Evaluation of the effectiveness and efficiency, (6) Communication. A two-stage iteration through the steps of the DSRM is aimed. The first iteration consists of steps 1-3 and will be part of this paper (see section 5). During the second iteration, the presented approach should be adapted according to new insights and finally prototypically developed, demonstrated and evaluated (steps 3-6). The results of the second iteration will be presented in another paper. Our first step of the DSRM, is based on expert interviews and a literature review. The aim of this step is to identify current problems in SCM regarding information exchange and to motivate the research question. Within the second step of the DSRM, the objective of a solution is defined from derived requirements along with the challenges and existing approaches from literature trying to fulfill them. Afterwards the gained insights were used to design a process for solving the problem as part of step three. The implementation (3) and execution of the demonstration step (4) as well as the evaluation (5) and communication (6) steps are planned in the near future. This paper concludes with an outlook on this future steps based on the results of this work. In the following subsections, the procedure of the first iteration will be described in more detail.

\subsection{Expert Interviews}

The aim of the expert interviews is to provide a realistic coverage of current problems for interorganizational information exchange in SCM and today's trend to address these issues using DLT, respectively Blockchains. In order to gain a broad insight into this topic, representatives of various companies and scientific institutions with correspondingly different backgrounds were selected and contacted. The spectrum ranges from founders of a Blockchain startup to representatives of an established enterprise software house, which provides Blockchain 
solutions in enterprise software. All experts had experience with the realization of Blockchain projects, either from an advisory or technological point of view. The interviews were conducted over the phone and lasted between thirty minutes and one and a half hours. Semi-structured interviews were used, in which all desired topics can be covered with the help of preformulated questions [16]. The interviews were conducted openly based on the guidelines of the questionnaire. First, general questions were asked about the person in order to be able to classify the background of the participants. This was followed by questions about the current state of supply chains, such as: Who are the different stakeholders within a supply chain? What information is currently exchanged between them and through which channels? Is there trust between them? What is the current IT infrastructure in companies for storing and transmitting data like and what are the problems? Subsequently, it was discussed how DLT can solve these problems and what advantages and disadvantages they have. Finally, the future of DLT solutions in general and in the context of the companies was discussed. A total of six expert interviews were carried out. The audio tracks of these interviews were recorded. Based on these records, a written protocol was prepared for further evaluations. With the help of additional literature in the context of Blockchain and DLT for interorganizational information exchange, problems were identified related to this topic in the SCM. Finally, the requirements could be derived from the previous findings.

\subsection{Literature Review}

The literature review is used as part of DSRM step two to gather knowledge about feasible objectives and requirements for a solution which goes beyond the findings from the expert interviews in SCM. To identify publications related to the sharing and valuation of information in a collaborative network, we searched in the following scientific databases to ensure a comprehensive coverage of the subject: IEEE Xplore, ACM Digital Library, ScienceDirect and EBSCOhost. The electronic databases were searched with the string "information AND value AND shar* AND collaborative network" in the title, abstract and keywords. We have explicitly excluded the search term "supply chain" to obtain a broader range of possible references in the context of different branches and use cases of CNs. That search returned a total of 400 results. In the first step, the authors filtered these results by analyzing the title, abstract and keywords regarding the problems identified in DSRM step one. Also 15 duplicates were sorted out here. If a minor relevance was identified, the publications were marked for a second, finer granular rating, where the full-texts were considered in detail. In total 70 publications were marked as relevant in the first step. The final collection of relevant articles included 15 publications after the finer granular rating in the second filtration step. To prevent the exclusion of key papers, forth and back references are considered in a last step so that a total of 18 papers were considered.

\section{Approach}

Within this section, we apply the described methodology to tackle the research challenge. In order to derive requirements, we describe the results of the conducted expert interviews, followed by related approaches from literature and our resulting process proposal. Based on the expert interviews and the combined literature review, seven organizational, two economical and four technical problems were identified in the context of information exchange in supply chains (see Figure 1).

\subsection{Requirements from Expert Interviews}

Following, requirements are derived from problems identified by the expert interviews and considered in more detail by corresponding literature. Supply chains tend to be complex and consist of a large number of participants, which are often solely interested in the profit and success of their own business [17]. An efficient collaboration is therefore difficult, because sometimes no sense of community is available $(\mathrm{Ol})$. The reason for this is not always the lack of cooperation, but cultural obstacles $(\mathrm{O} 2)$ arising from the different origins of the companies $[18,19,20]$. In order to solve these problems and overcome the obstacles, a rethinking of a cooperative mindset must take place [18,19]. A platform cannot do that. For this reason, the psychological barrier to participation in a common solution must be kept as low as possible $(R l)$.

Based on the experts' statements, the supply chain is not only complex, but also intransparent. Companies often only know their direct contacts or a small number of their downstream and upstream contacts [17]. For example in the food industry, participating companies in a supply chain are only required to store information about the companies from which products have been received ("one-step-down") and information about the companies to which the products have been delivered ("one-step-up") [21]. Product recalls in the food industry are complicated and costly, because along the supply chain the cause of the defect must be determined systematically over the different connections [22]. Not only have the companies themselves lacked transparency, but also the consumer. The consumer 
desire for information on qualitative characteristics e.g. in the food supply chain the need for proof of origin or bio-credentials is increasing and therefore also the need of a transparent solution [22]. A common solution must resolve the lack of transparency (O5) by satisfying both information providers and the information consumers with the degree of information (RO).

Due to the large number of participants in a supply chain, the management and planning of collaborations are not easy and often led to coordination problems (O8) $[23,24]$. Currently, finding a provider for a specific job is costly and time-consuming because of the lack of transparency [17]. A common platform must keep the coordination effort low $(R 7)$.

One of the main problems is the lack of trust (O4) $[23,24,25]$. A manager of an enterprise software house reports that when implementing a common Blockchain solution to optimize the food supply chain, the various stakeholders do not want to be identifiable because of the fear that they could be passed over and replaced by a new stakeholder. The trust that the information will not fall into the wrong hands is important for a cooperative solution, especially when sensitive information needs to be shared [23]. With the help of the expert interviews, the following types of information were identified as sensitive information, which companies are reluctant to share with other stakeholders: (1) identifying business partner data and supplier information; (2) order and billing information e.g. prices, quantities; (3) company secrets e.g. recipes. Accordingly, as a requirement for a common solution, it is inferred that participants should retain control over their information resources and that the degree of information exchange should be determinable $(R 2, R 3)$. In addition to trusting the other participants, there must also be some trust in the technology [25]. Only in this way it can be ensured that participants do not withhold information, despite their own control over the degree of information exchange. Withholding information can reduce the effectiveness of a common solution for information exchange [23]. The trust in the technology is strengthened in particular by the aspects robustness, reliability and security [19]. Therefore the common solution should provide a secure and confidential channel for sharing information over a neutral decentralized platform $(R 4, R 5)$. According to the experts, power structures often prevail (O3) in supply chains. These structures mean that stakeholders which are more powerful are compelling the other less powerful stakeholders to join their intended platform [26]. This approach is not conducive to building trust [27]. In a common solution in which such power relations do not predominate, the powerful participants are afraid that they will lose the control over the projects [28]. As a result, the psychological barrier for participation should be low $(R l)$. No matter how technologically mature, secure and trustworthy a common solution may be, it is still useless if nobody participates in it. For this reason, an additional benefit must be recognizable for each participant. The problem here is that the additional value is not obvious for each participant $(E 1)$ in the supply chain, because there is not always a monetary advantage $[18,19,26]$. Why, for example, should a farmer take part in a common solution for information exchange and spend his time writing down information that might give others of the supply chain a monetary advantage? Especially, if he has to buy hardware for the information input and therefore even has monetarily disadvantages? "At this point, it is necessary to be persuasive, since these actors are nevertheless important information providers in the network" (developer of an enterprise software house). Even if the various stakeholders can be motivated to participate, there is the problem of having to clarify which stakeholders finance the common solution. Due to the lack of a cooperative mindset, according to the experts it is possible that this point will lead to disagreements over the financing (E2), because often the responsibility for paying costs is shifted between the companies [24]. For a common solution, this means that both the costs for the participation and the profits earned collectively through the information exchange must be fairly distributed so that everyone benefits and has an incentive to participate $(R 10)$.

In addition to the aforementioned organizational problems, the implementation of a common technology also raises technical barriers, which need to be overcome. In recent years, companies have streamlined the exchange of information within the enterprises so that employees have access to consistent data through databases or cloud services [29]. A manager in the Blockchain section of an enterprise software house mentioned in the interview that beyond the organizational boundaries, there is no real-time exchange of information (T4). He describes the current process of interorganizational information exchange as follows: "You send an order via FAX/EDI/XML Document/RosettaNet/Web Service to your business partner and hope that someday he will answer. This has nothing to do with real time anymore. You may receive a confirmation in real time that the document has been received". Accordingly, a common solution would require a platform that allows the real-time information sharing, so that everyone can work on the same data, not on erroneous and outdated data (R17). Another technical problem is that every company has different technological capabilities (T3) for the exchange of information [24]. Looking back at the farmer in the food supply chain, he will presumably have different hardware specifications or channels for information 
exchange than a large retailer. According to the experts, the exchange of information is sometimes still offline using paper. For this reason, a common solution must be set up so that a small minimum technical requirement is needed for participation and digitization of the information (R16). Because of these different capabilities and lack of compatibility between the different systems, interoperability is also hard to achieve. The reasons for the lack of interoperability (T2) are usually missing standards $[24,28]$. In addition to the hardware standards, there are also missing standards for the exchange of information, which specify in which format information should be exchanged [23,28]. To improve interoperability, industry and data standards are needed [18]. Because of this, a standardized solution is preferred in a common platform for interorganizational information exchange (R14).

Due to a lack of interoperability, producers, for example, tend to make their own platforms available for the exchange of information with the standards they have chosen. Suppliers are forced to use all different platforms and must adapt to each producer's standards, which is costly and time-consuming $[26,30]$. To ensure interoperability, a common solution should avoid a socalled vendor lock-in (R15) [30].

\subsection{Approaches from Literature Review}

Most found publications deal with theoretical models to simulate CNs and the dynamics prevailing in such environments to capture the value of information sharing. Prominent examples for such models are the one proposed by Fiala et al. [31] and the model of Cachon et al. [32]. Fiala et al. models the flow of material, information and finance in a supply chain. Cachon et al. examines the added value of sharing inventory information in a supply chain and compares the costs obtained by this approach with the costs of systems that follow traditional policies. In their study, costs have been reduced by $2.2 \%$ by sharing information. Both showed that information sharing can be useful in supply chains to reduce the bullwhip effect [31,32]. Chituc et al. [33] define performance metrics to measure the performance assessment of a CN. According to them, the decision whether to join, leave or remain in a $\mathrm{CN}$ depends on the three defined performance metrics: costs, payoff and agility. They also demonstrate analytical models to estimate them $[33,34]$. Based on their research, it can be deducted that an incentive to participate in a $\mathrm{CN}$ is the prior clarification of the join/leave/remain problem by contextualizing the three metrics. A possible solution should support the decision-making process by considering metrics as requirement $(R 11)$. In Benqatla et al. [35] a similar approach is modeled with the help of the actor network theory in order to motivate actors to cooperate in CNs by calculating cost-savings related to the participation. This model together with the research of Susha et al. [12] supports the expert statements that a situation is needed where every participant benefits from a common solution (R10). Susha et al. notes that the fear of competitive advantage by other organizations could be an obstacle of extended information sharing. Pardo et al. [36] argues that between the members of a $\mathrm{CN}$, trust, mutuality and a common identity should preveal. According to Fulford et al. [37] especially small enterprises cannot fully exploit the potential for collaboration, as they do not have the resources to build up systems to allow collaboration. To counteract this, the psychological and technical barriers for participation in a $\mathrm{CN}$ should be low $(R 1, R 16)$. A further aspect that is mentioned by Woods et al. [38] and Freudiger et al. [39] is the consideration of the quality of the shared data. In order to prevent frustrations regarding different data qualities, Woods et al. propose a data mining method to structure the shared data (T1). Persistent repositories for the storage of shared data are proposed as relevant (R13). Freudiger et al. on the other hand, uses protocols so that an organization can test the data quality of shared data provided by a server prior to the purchasing of the data. In order to protect data privacy, only the value of the quality metrics are shared. Especially in the context of data monetization they see the ability to check the data quality prior as very useful (R12). In the context of information markets, Vishik et al. [40] see also recognizable currencies as more efficient than pure data exchange models, especially because of the challenge of reconciling different options and views on the value of information and the reduced search effort. According to them, these currencies do not necessarily have to be of monetary nature and can also be tokens. Fleisch et al. [11] mention that the classical supply chains are replaced by more complex, flexible and temporary $\mathrm{CNs}$, which require more skills from the information manager $(O 7)$. In order to facilitate the coordination and sharing of information in such networks, we believe that a possible solution should support the decision-making of information managers $(R 9)$.

Beside theoretical models, studies and reviews in the context of information sharing and coordination have been found. Durugbo [10] investigated in a case study to what extent $\mathrm{CNs}$ can be used for the management of integrated information flows and proposed a conceptual framework to manage the flow integration. They propose the need for $\mathrm{CN}$ managers to prevent vague collaborative agreements, generate procedurally prompts, make implementation checklists, strengthen the relationship and trust with partners, use systematic templates for communication, determine stern issuance 
policies, as well as the separation of business and technological concerns over the collaboration time. For this purpose they also suggest to build up a decision support system for $\mathrm{CN}$ manager $(R 9)$. Günther et al. [41] has conducted a case study in the timber industry to examine how an implemented supply chain management system can support collaborative planning processes but could't find noticeable improvements. In their opinion, this may be due to the fact that the users of the system have not been sufficiently trained to use it and have therefore lost their motivation. For this the psychological and technological barriers to participate should be low $(R 1, R 16)$. In the study of Brown et al. [42], the technological and organizational challenges of sharing cyber security information are presented and requirements from the community are summarized for a possible solution. The key challenges they mentioned for building such systems are: working with multiple information sources, combining, determining and enriching data and the allocation of the information into organizational workflows and technological products (R14). No technological solutions to these problems are mentioned.

Also existing technological implementations could be identified with the help of the literature review. According to Kadar et al. [43], negotiations are the basis for cooperation and coordination between actors. They use a multi-agent based negotiation system to maintain sustainable interoperability. The decentralized negotiation process that is mapped by the system represents contract negotiations between organizations where dissatisfaction with the current process leads to disruption of interoperability and thus to renegotiation. Based on their research, we believe that a decentralized negotiation or voting system can be useful for the coordination of information sharing in CNs as well $(R 8)$. The literature review also identified a publication in the field of DLT, namely that of Angrish et al. [44]. They use a decentralized approach to handle manufacturing information generated by machines and computing nodes of different organizations using Blockchain technology. The focus here is on the design of the computing nodes and the physical devices and the connection of these via the Blockchain. No possibility for the systematic coordination of the information is shown. But they also emphasize that organizations must find ways to collaborate and share information in an inherently untrusted network. A similar approach is used by Pouly et al. [45]. They present a method for the automatic collection of manufacturing information, which forwards them automatically to the ERP system of an organization. These ERP systems are in turn interconnected to a central data warehouse.

Based on the described results, it can be concluded that the literature review did not identify a systematic approach for the coordination of the search process which fulfills the derived requirements from the expert interviews. Especially with the new capabilities of DLT, we propose to come a step closer to solving the problem. For this purpose the following process is inspired by the problems and requirements of the mentioned approaches from literature combined with the insights from the expert interviews.

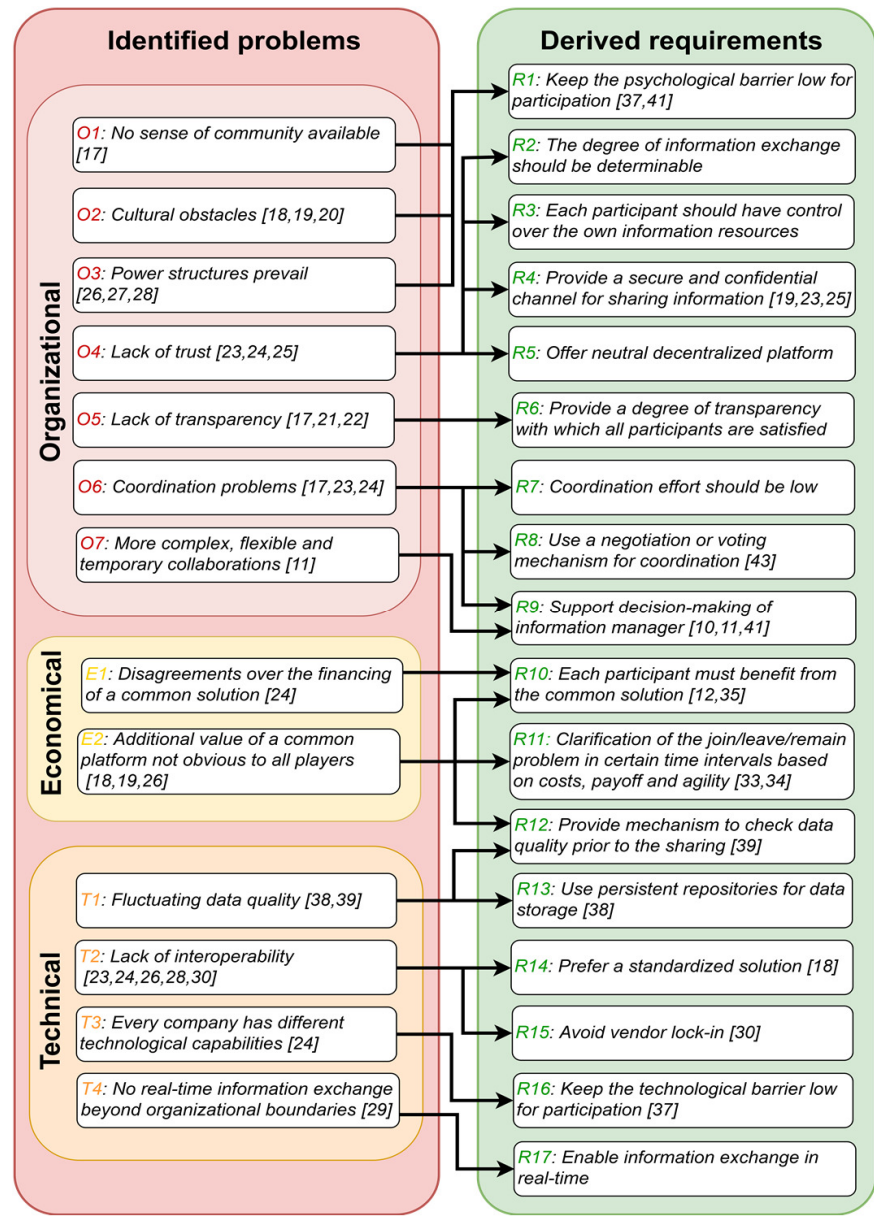

Figure 1: Identified problems and derived requirements [17-27]

\subsection{Proposed Process and System Components}

In the previous section, we identified the need and prerequisites to reduce information asymmetry in CNs. Therein, convincing all relevant network partners to resolve asymmetric information by sharing sensitive data is a challenge. To face this systematically, we model an abstract search process to coordinate the finding of a win-win situation as solution artifact following step three of the DSRM (see figure 2). Our proposed process consists of four phases as well as several tasks in a chronological order to record information needs, their dependencies and value. The 
developed phases and mechanisms as well as how they could be implemented according to the requirements mentioned above (see section 5.1) are described below to put it up to discussion.

We hypothesize that DLT as it is proposed for information sharing in literature could also act as an integrative force between organizations for such coordination processes. Alternatively an intermediary has to address the described challenge, which contradicts the requirements derived from our studies $(R 1, R 5, R 11, R 15)$. That's why we propose a permissioned DLT-backed system design.

According to our observations in the SCM, we identify application-specific optimization requests, as triggering events such as product recalls or reducing bullwhip effects. As prerequisite, the initiating partner who identified optimization potential should invite the other partners to join a coordination process for its request. These events cause a search for information that may be provided by other partners but is currently not available for various reasons (see section 5.1). Therefore it should state the reason and target for his request to give other partners in the network a hint of possibly needed information and an incentive to take part of his initiative $(R I)$. To limit the process in duration and reach the optimization target in finite time, three dates have to be defined in chronological order (Date $1<$ Date $2<$ Date 3 ) and communicated by the initiating partner as stop criterion for the first three phases of the process. Also a predefined number of iterations per phase is conceivable to give the chance to adapt during the process phases. How to choose feasible timeframes and numbers of iterations has to be determined heuristically.

Phase 1: Estimate Costs \& Identify Information Needs and their Value Proposition

To keep coordination effort low, this phase could be done informally e.g. via email and manually managed tools by each partner (R7). As our investigation showed, in SCM generally a one-step-forward and one-stepbackward relationship exists between partners with a certain level of trust. Therefore, the initiating request event must be forwarded by the partners to the respective other participants along this chain of trust (R7) in the network until Date 1. In order to overcome this hurdle, the initiating partner might work with incentives like data offerings in advance $(R 1)$. Also data sets to gain an impression about data quality may increase willingness to participate (R12). As support for a common understanding and as a template for the identification of the relevant information artifacts (ontology of data source and its meta data like content, format, access point, etc. which has to be defined after further investigation), definitions of standardized industry-based communication protocols might be used
$(R 2, R 14, R 17)$. In parallel, each partner should also estimate costs and value propositions in order to determine metrics for evaluating the potential participation in information sharing (R11).

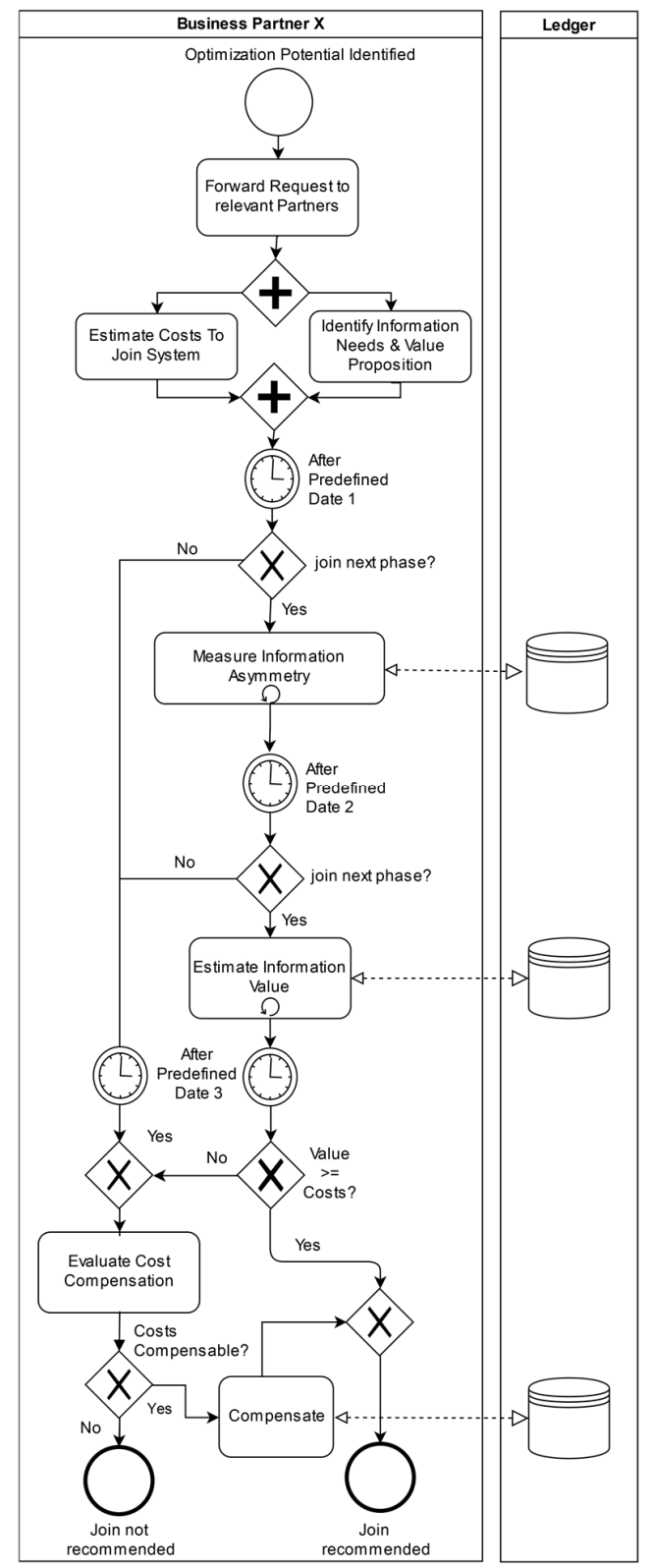

Figure 2: Derived abstract process model

Phase 2: Measure Information Asymmetry

As a next step, we consider how the interest in information in the network is distributed and to what extent these interests depend on each other is part of this phase whereby an agreement about needed information could be accomplished. This should make transparent which information is relevant in the network and how the respective partners evaluate its priority. 
From a processual point of view, the information artifacts identified in Phase 1 must first be completely weighted by the respective partners until Date 2. Prioritization can be specified by using simple scoring mechanisms. For example, every participant has 100 points and can thus add and evaluate the information artifacts of interest over the predefined number of iterations until Date 2. Due to the desired level of transparency, scoring can also be blind during the voting phase as not to be influenced by each other but subsequently disclosed. As results of this voting phase, preference relations about the information artifacts within the network, the importance of single partners in this setting and strength of relationships between them can be determined. Therefore, the initiating partner has to make sure that partners who are willing to join are able to participate in Phase 2. Also, declining partners should be remembered because they could be important for compensation evaluation in Phase 4.

From a technological point of view, we propose a voting system, that can be operated independently of a trusted instance regarding the derived requirements due to a missing sense of community as well as lack of trust and transparency $(R 1, R 4, R 5)$. We propose a DLT-based voting system $(R 8)$ to share the operation expenses $(R 6$, $R 10$ ) for specifying and prioritizing the required information artifact. Voting can be performed using transactions of tokens in a distributed ledger as proposed in literature $[2,46]$. Additionally blind voting could be possible, where scores can be submitted pseudonymous and encrypt on-chain data [2] until specified Date 2.

\section{Phase 3: Estimate Information Value}

After relevant information artifacts are identified in Phase 2, a mechanism is needed to help the partners assessing the potential value of their data for decisionmaking about information sharing collaborations $(R 9)$. A market-oriented approach is proposed to approximate the values. The valuation of information is a field of research for years and is exposed to many challenges [47]. The trading of data and the development of data marketplaces are also associated with numerous open questions that have already been discussed in research [48]. We do not claim to solve the challenges in the research fields described above. Our approach is based on the assumption that a market-driven approximation of information value can be controlled by the demand of the partners. This demand is subject to an idea of optimization, from which the partners expect a benefit, which we will try to quantify here as a contribution to the base for decision-making.

In this phase, we also consider a DLT-based information system component to be advantageous for the implementation of an auction mechanism $(R 8)$. Here, we propose hashing the information requests consisting of e.g. price and information artifact as well as writing it as time-stamped transaction into a ledger before submitting it. This helps to coordinate requests and make them verifiable afterwards. The DLT component would then be used according to the reverse oracle pattern for Proof of Existence [2]. The requests can afterwards be disclosed to the respective addressee (R5), who could accept one of the offers (probably the highest) for itself or make a counter-offer to the requestor using the same logic. Under the assumption that the price only turns out after an iterative approximation, this evaluation process can be carried out over several rounds up to predefined Date 3, whereby a suitable information value can be determined for our basic idea.

\section{Phase 4: Consensus}

Finally, each partner can compare the value of its information artifacts with the effort it would take to participate in a collaborative system. An agreement could be accepted if the previously estimated costs of participation in the network per partner is less than the value of salable information and estimated value proposition of needed information. If no agreement can be found between individual partners or the network depends on declining partners from earlier phases, compensation mechanisms should also be discussed based on the dependency relationships established in Phase 2 (R2). A DLT-based system that can achieve compensation between partners via tokenization pattern [2] in the form of a cryptocurrency could also be suitable for this purpose. Herein, there are also further challenges to discuss, in particular the danger of fraud, which is why this step must be considered with separate research efforts in the future $(R 3)$. The prospect of a win-win situation could outweigh the fear of fraud and can serve as motivation for a functioning compensation mechanism.

\section{Results \& Limitations}

In this work, we analyzed problems and requirements regarding interorganizational information exchange in CNs to understand the potential role DLT could play. By conducting semi-structured expert interviews and literature review, we derived the need to systematically investigate information asymmetry in CNs. Combined with insights from an extensive literature review, we developed a search process for a win-win situation which is aimed to identify the degree of information asymmetry and potential value of information exchange. Using existing DLT design patterns, we propose system components to coordinate this process without additional intermediaries [2]. Our approach is intended to overcome limited trust between partners and 
support the decision whether to establish a more data intense collaboration or not.

Due to the small number of participants in the expert interviews, we would like to emphasize that our investigations are not free of methodological limitations. Currently, there is only a small number of experts which implement Blockchain or related DLT solutions in SCM and are willing to talk about their experiences. Due to this fact, technology providers, consultants, entrepreneurs and researchers have been interviewed. These have mostly a positive attitude towards DLT due to their proximity to the technology and its value proposition. We have tried to compensate for these limitations by reviewing the literature to substantiate the derived requirements. We conducted our literature reviews to the best of our knowledge, but also see these processes as error-prone due to the choice of search terms, strategy and scientific databases. In addition, the derived process has limitations inherent. As discussed, the pricing of information and trading of data as well as several dangers of fraud, attacks and competitive analyses are separate research fields and must be considered with effort in the future to make this process practically useful.

\section{Future Work}

As a next step, we will take our second iteration of the DSRM. Therefore, the presented approach will be adapted according to new insights and game theoretic considerations. Finally, the system components can be prototypically implemented and evaluated with real world scenarios. To this end, information artifacts have to be modelled. Our goal is to build an open source tool $(R 15, R 16)$, which is easy to use, also for information managers in small businesses e.g. by being compatible with Blockchain as a Service (BaaS) provisioning. Furthermore, we can imagine the adoption in other areas than SCM, e.g. healthcare seems to be promising with similar problems. Additionally, upstream and downstream processes, which could be linked with the presented process, as well as the mentioned limitations, have to be further investigated and reduced in future.

\section{References}

[1] S. Nakamoto, "Bitcoin: A peer-to-peer electronic cash system", 2008.

[2] X. Xu,et al., "Architecture for Blockchain Applications", Springer International Publishing, 2019.

[3] S. Saberi et. al., "Blockchain technology and its relationships to sustainable supply chain management", International Journal of Production Research, 2019.
[4] S. van Engelenburg et al., "A Blockchain Architecture for Reducing the Bullwhip Effect", Business Modeling and Software Design, Springer, Cham, 2018.

[5] J. Mendling et. al., "Blockchains for business process management - challenges and opportunities", ACM TMIS, 2018.

[6] M. Iansiti and K.R. Lakhani, "The truth about blockchain", Harvard Business Review, 2017.

[7] N. El Ioini and C. Pahl, "A Review of Distributed Ledger Technologies", OTM Confederated International Conferences, Springer, 2018.

[8] M.S. Ali and K. Dolui, "IoT data privacy via blockchains and IPFS", Proceedings of the 7th IoT, Linz, Austria, 2017.

[9] Camarinha-Matos, Luis M. and Afsarmanesh, Hamideh, "Collaborative networks: a new scientific discipline". Journal of Intelligent Manufacturing 16 (4-5), 2005.

[10] C. Durugbo, "Managing information for collaborative networks", Industrial Management \& Data Systems, vol.114, no.8, 2014.

[11] E. Fleisch and S.G. Powell, "The value of information in a business network”, HICSS, IEEE, Maui, HI, USA, 2011.

[12] I. Susha et al., "Identifying Mechanisms for Achieving Voluntary Data Sharing in Cross-sector Partnerships for Public Good", dg.o 2019, ACM, Dubai, United Arab Emirates, 2019.

[13] K. Peffers, et al., "A Design Science Research Methodology for Information Systems Research", Journal of Management Information Systems, M. E. Sharpe, Inc., Armonk, NY, USA, 2008.

[14] A. Hevner et al., "Design Science In Information Systems Research", MIS Quarterly Vol. 28 No.1, SIM and MIS Minneapolis, USA, 2004.

[15] V. Vaishnavi and W. Kuechler, "Design Science Research Methods and Patterns: Innovating Information and Communication Technology", Auerbach Publications Boston, Boston, MA, USA, 2008.

[16] H.J. Rubin and I.S. Rubin, "Qualitative Interviewing: The Art of Hearing Data", SAGE Publications, Thousand Oaks, CA, USA, 2012.

[17] Y. Fu and J.Zhu, "Big Production Enterprise Supply Chain Endogenous Risk Management Based on Blockchain", IEEE Access, IEEE, 2019.

[18] C. Hsu et al. "A legitimacy challenge of a cross-cultural interorganizational information system", European Journal of Information Systems, 2015.

[19] C.M. Messerschmidt and O. Hinz, "Explaining the 
adoption of grid computing: An integrated institutional theory and organizational capability approach", Journal of Strategic Information Systems, 2013.

[20] M.L. Tushman and C. O'Reilly, “Ambidextrous Organizations: Managing Evolutionary and Revolutionary Change”, California Management Review, 1996.

[21] L. Ruiz-Garcia, G. Steinberger and M. Rothmund, "A model and prototype implementation for tracking and tracing agricultural batch products along the food chain", Food Control, 2010.

[22] S. Al Tal, "Modeling information asymmetry mitigation through food traceability systems using partial least squares", Electronic Journal of Applied Statistical Analysis, 2012.

[23] C. Van Slyke et al., "Overcoming barriers to distributed interorganizational systems”, HICSS, IEEE, USA, 1997.

[24] K. Zhu et. al., "Migration to Open-Standard Interorganizational Systems: Network Effects, Switching Costs and Path Dependency”, MIS Quarterly, 2006.

[25] T. Verhagen et al., "Perceived risk and trust associated with purchasing at electronic marketplaces", European Journal of Information Systems, 2006.

[26] J. Webster, "Networks of collaboration or conflict? Electronic data interchange and power in the supply chain", The Journal of Strategic Information Systems, 1995.

[27] H. Teo et al., "Prediction Intention to Adopt Interorganizational Linkages: An Institutional Perspective", MIS Quarterly, 2003.

[28] M. Hardwick et. al., "Sharing Manufacturing Information in Virtual Enterprises", Commun. ACM, New York NY (USA), 1996.

[29] A. Khajeh-Hosseini et al., "Cloud Migration: A Case Study of Migrating an Enterprise IT System to IaaS", CLOUD, IEEE, 2010.

[30] J. Opara-Martins et al., "Critical analysis of vendor lockin and its impact on cloud computing migration: a business perspective", Journal of Cloud Computing: Advances, Systems and Applications, 2016.

[31] P. Fiala, "Information sharing in supply chains", Omega, vol. 33, no. $5,2005$.

[32] G. P. Cachon and M. Fisher, "Supply Chain Inventory Management and the Value of Shared Information", Manager Science, vol.46, no.8, 2000.

[33] C.-M. Chituc et al., "The JLR decision model for CNOs: Payoff criteria extension", IFAC Proceedings Volumes, vol. 40, 2007.

[34] C.-M. Chituc and S.Y. Nof, "The Join/Leave/Remain
(JLR) decision in collaborative networked organizations", Computers \& Industrial Engineering, vol. 53, 2007.

[35] M. S, Benqatla et al., "Actor network theory as a collaborative mode: The contribution of game theory in the interessement phase", ICICT, IEEE, Marrakech, Morocco, 2016.

[36] T.A. Pardo et al., "Governance Structures in Cross Boundary Information Sharing: Lessons from State and Local Criminal Justice Initiatives", HICSS, IEEE, Waikoloa, HI, USA, 2008.

[37] R. Fulford and C. Standing, "Construction industry productivity and the potential for collaborative practice", International Journal of Project Management, vol. 32, 2014.

[38] B. Woods et al., "Data Mining for Efficient Collaborative Information Discovery", Proceedings of the 2nd ACM Workshop on Information Sharing and Collaborative Security, ACM, Denver, Colorado, USA, 2015.

[39] J. Freudiger et. al., "Privacy Preserving Data Quality Assessment for High-Fidelity Data Sharing", Proceedings of the 2014 ACM WISCS, Scottsdale, Arizona, USA, 2014.

[40] C. Vishik and A.B. Whinston, "Knowledge Sharing, Quality, and Intermediation", Proceedings of the WACC, ACM, 1999.

[41] H. Günter et al., "Information technology in supply networks: Does it lead to better collaborative planning?", Journal of Enterprise Information Management, vol. 19, 2006.

[42] S. Brown et al., "From Cyber Security Information Sharing to Threat Management", WISCS, ACM, Denver, Colorado, USA, 2015.

[43] M. Kadar et. al. “A Multi-agent Based Negotiation System for Re-establishing Enterprise Interoperability in Collaborative Networked Environments", ICCMS, IEEE, Cambridge, UK, 2013.

[44] A. Angrish et. al., "A Case Study for Blockchain in Manufacturing: "FabRec": A Prototype for Peer-to-Peer Network of Manufacturing Nodes", Procedia Manufacturing, vol. $26,2018$.

[45] M. Pouly and S. Berthold, "Digital factory for collaborative supply chains", CSCWD, IEEE, Shanghai, China, 2010

[46] F.P. Hjálmarsson et al., "Blockchain-Based E-Voting System", CLOUD, IEEE, San Francisco, CA, 2018.

[47] J.-P. Chavas and R. D. Pope. "Information: Its Measurement and Valuation", American Journal of Agricultural Economics, 1984.

[48] F. Schomm, F. Stahl and G. Vossen, "Marketplaces for data: an initial survey", ACM SIGMOD Record, 2013. 